\title{
A NEW NEUTROSOPHICAPPRAOCH TO IMAGE THRESHOLDING
}

\author{
Yanhui Guo $^{1,2}$, H.D. Cheng ${ }^{1,2}$, Yingtao Zhang' ${ }^{1}$, Wei Zhao ${ }^{1}$ \\ ${ }^{1}$ School of Computer Science and technology, Harbin Institute of Technology, Harbin, \\ China, 150001 \\ ${ }^{2}$ Department of Computer Science, Utah State University, Logan, UT 84322 U.S.A.
}

\begin{abstract}
A neutrosophic set (Ns), a part of neutrosophy theory, studies the origin, nature, and scope of neutralities, as well as their interactions with different ideational spectra. The neutrosophic set is a powerful general formal framework that has been recently proposed. However, the neutrosophic set needs to be specified from a technical point of view. We apply the neutrosophic set in image domain and define some concepts and operations for image thresholding.

The image $G$ is transformed into Ns domain, which is described using three subsets $T, I$ and $F$. The entropy in neutrosophic set is defined and employed to evaluate the indetermination. A new $\lambda$ mean operation is proposed to reduce the set's indetermination. Finally, the proposed method is employed to perform image thresholding. We have conducted experiments on a variety of images. The experimental results demonstrate that the proposed approach can select the thresholds automatically and effectively. Especially, it can process the "clean" images, the images with different kind of noise and the images with multiple kinds of noise well without knowing the type of the noise, which is the most difficult task for image thresholding.
\end{abstract}

Keywords: Neutrsophic set, Maximum entropy, Image threshold.

\section{Introduction}

Neutrosophy, a branch of philosophy, introduced by Florentin Smarandache [1] as a generalization of dialectics, which studies the origin, nature, and scope of neutralities, as well as their interactions with different ideational spectra.

Neutrosophy theory considers every proposition, theory, event, concept, or entity, $<\mathrm{A}>$ in relation to its opposite, $<$ Anti-A $>$ and the neutralities $<$ Neut-A $>$, which is not $\mathrm{A},<$ Non-A $>$, and that which is neither $\langle$ A $>$ nor $\langle$ Anti-A $>$. The $<$ Neut$\mathrm{A}>$ and $<$ Anti-A $>$ are referred to as $<$ Non-A $>$. According to this theory every idea $<A>$ tends to be neutralized and balanced by $<$ Anti-A $>$ and $<$ Non-A $>$ ideas.

In a classical way $\langle$ A $>$, $\langle$ Neut-A $>$, $<$ Anti-A $>$ are disjoint two by two. In many cases the borders between notions are vague, imprecise, and it is possible that $<$ A $>,<$ Neut-A $>,<$ Anti-A $>$ (and $<$ Non-A $>$ of course) have common parts two by two as well.

Neutrosophy is the basis of neutrosophic logic, neutrosophic probability, neutrosophic set, and neutrosophic statistics [1].

For classical set, the indetermination of each element in the set could not be evaluated and described. The fuzzy set introduced by Zadeh [2] has been applied in many real applications to handle uncertainty. The traditional fuzzy set uses a real number $\mu_{A}(x) \in[0,1]$ to represent

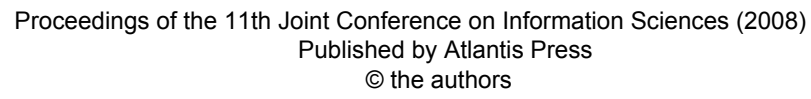


the membership of fuzzy set $A$ defined on universe $X$. Sometimes, if $\mu_{A}(x)$ itself is uncertain, it is hard to be defined by a crisp value [3]. In some applications such as expert system, belief system and information fusion, we should consider not only the truth-membership supported by the evidence, but also the falsity membership against by the evidence. That is beyond the scope of fuzzy sets.

In neutrosophic set, indeterminacy is quantified explicitly and the truthmembership, indeterminacy-membership and falsity-membership are independent. This assumption is very important in many applications such as information fusion in which we try to combine the data from different sensors. Neutrosophic set generalizes the concept of the classic set, fuzzy set [2], interval valued fuzzy set [4], intuitionistic fuzzy set [5], interval valued intuitionistic fuzzy set [6], paraconsistent set [1], dialetheist set [1], paradoxist set [1], tautological set [1].

In this paper, the new Neutrosphic set approach to image thresholding is proposed. First, the image is transformed into neutrosophic set and a new operation, $\lambda$-mean, is employed to reduce the indetermination degree of the image, which is evaluated by the entropy of the indeterminate subset. Then, the image becomes more uniform and homogenous, and more suitable for thresholding. Finally, the image in Ns is thresholded using a histogram threholding method. The experiments on artificial images with different levels of noise and real images demonstrate that the proposed approach can reduce the indeterminate of the images and perform thresholding with better result especially, in noisy cases.

\section{Proposed method}

\subsection{Neutrosphic set}

Neutrosophic set and its properties are discussed briefly [1].

Definition 1 (Neutrosophic set) Let $U$ be a universe of discourse, and a neutrosophic set $A$ is included in $U$. An element $x$ in the set $M$ is noted as $x(T, I, F) . T, I$ and $F$ are real standard or non-standard subsets of $]^{-} 0,1^{+}[$ with $\sup T=t_{-}$sup , inf $T=t_{-}$inf , sup $I=i \_s u p \quad, \quad$ inf $I=i \_i n f$, sup $F=f \_s u p \quad, \quad$ inf $F=f \_i n f$ and $n \_$sup $=t \_s u p+i \_s u p+f \_s u p$ $n \_$inf $=t \_i n f+i \_i n f+f \_$inf $. T, I$ and $F$ are called neutrosophic components.

The element $x(T, I, F)$ belongs to $A$ in the following way: it is $t \%$ true in the set, $i \%$ indeterminate in the set, and $f \%$ false, where $t$ varies in $T, i$ varies in $I$, $f$ varies in $F$. Statically $T, I$ and $F$ are subsets, but dynamically $T, I$ and $F$ are functions/operators depending on many known or unknown parameters. The subsets $T, I$ and $F$ are not necessarily intervals, and may be any real subunitary subsets: discrete or continuous; single-element, finite, or countable or uncountable infinite; union or intersection of various subsets; etc. They may also overlap.

In neutrosophic set, some basic operations are defined accordingly.

\subsection{Neutrsophic Image}

A new neutrsophic approach to image processing is discussed.

Definition 2 (Neutrosophic image): Let $U$ be a universe of discourse, and $W$ be a set included in $U$, which is composed by bright pixels. A neutrosophic image $P_{N s}$ is characterized by three subset $T, I$ and $F$.

A pixel $P$ in the image is described as $P(T, I, F)$ and belongs to $W$ in the following way: it is $t \%$ true in the bright

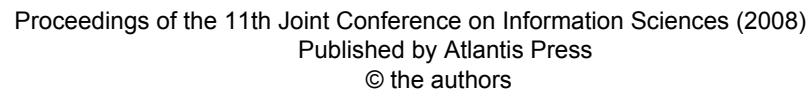


pixel set, $i \%$ indeterminate, and $f \%$ false, where $t$ varies in $T, i$ varies in $I$, and $f$ varies in $F$.

The pixel $P(i, j)$ in the image domain is transformed into neutrosophic domain $P_{N S}(i, j)=\{T(i, j), I(i, j), F(i, j)\}$

$T(i, j), I(i, j)$ and $F(i, j)$ are the probabilities belong to white set, indeterminate set and non-white set, respectively, which are defined as:

$$
\begin{aligned}
& T(i, j)=\frac{g(i, j)-g_{\min }}{g_{\max }-g_{\min }} \\
& I(i, j)=1-\frac{H o(i, j)-H o_{\min }}{H o_{\max }-H o_{\min }} \\
& F(i, j)=1-T(i, j) \\
& H o(i, j)=\operatorname{abs}(e(i, j))
\end{aligned}
$$

where $H o(i, j)$ is the homogeneity value of $T$ at $(i, j)$, which is described by the local gradient value $e(i, j)$.

After the image is transformed in Ns domain, some concepts and operations in neutrosophic domain are defined and employed.

\subsection{Neutrosophic Image Entropy}

For gray image, entropy is utilized to evaluate the distribution of different gray level. If the entropy is maximum, the different intensities have equal probability and the intensities distribute uniformly. If the entropy is small, the intensities have different probabilities and their distributions are non-uniform.

Definition 3 (Neutrosophic image entropy) Neutrosphic image entropy is defined as the summation of the entropies of three subset $T, I$ and $F$, which is employed to evaluate the distribution of the elements in neutrosphic domain:

$$
\begin{aligned}
& E n_{N S}=E n_{T}+E n_{I}+E n_{F} \text { (5) } \\
& E n_{T}=-\sum p_{T}(i) \ln p_{T}(i)(6) \\
& E n_{I}=-\sum p_{I}(i) \ln p_{I}(i)
\end{aligned}
$$

$$
E n_{F}=-\sum p_{F}(i) \ln p_{F}(i)
$$

where $E n_{T}, E n_{I}$ and $E n_{F}$ are the entropy of subsets $T, I$ and $F$, respectively. $p_{T}(i), p_{I}(i)$ and $p_{F}(i)$ are the probabilities of element $i$ in $T, I$ and $F$, respectively. $E n_{T}$ and $E n_{F}$ are utilized to measure the distribution of the elements in Neutrosophic set, and the entropy of $T, E n_{I}$ is employed to evaluate the indetermination distribution.

\section{4. $\lambda$-mean operation}

The value of $I(i, j)$ is employed to measure the indeterminate degree of element $P_{N S}(i, j)$. It means that the element is indeterminate, whose indeterminate degree is measured by the value in I subset. For making subset $I$ be correlated with $T$ and $F$ subsets, the changes in $T$ and $F$ influence the distribution of element in $I$ and the entropy of $I$.

In gray level domain, a mean operation for image $X$ is defined as:

$$
\bar{X}(i, j)=\frac{1}{w \times w} \sum_{m=i-w / 2}^{i+w / 2} \sum_{n=j-w / 2}^{j+w / 2} X(m, n)
$$

Definition 8 ( $\lambda$-mean operation): A $\lambda$-mean operation for $P_{N S}, \bar{P}_{N S}(\lambda)$ is defined as:

$$
\begin{array}{r}
\bar{P}_{N S}(\lambda)=P(\bar{T}(\lambda), \bar{I}(\lambda), \bar{F}(\lambda))(10) \\
\bar{T}(\lambda)=\left\{\begin{array}{ll}
T & I<\lambda \\
\bar{T} & I \geq \lambda
\end{array}(11)\right. \\
\bar{T}_{\lambda}(i, j)=\frac{1}{w \times w} \sum_{m=i-w / 2}^{i+w / 2} \sum_{n=j-w / 2}^{j+w / 2} T(m, n) \\
\bar{F}(\lambda)=\left\{\begin{array}{ll}
F & I<\lambda \\
\bar{F} & I \geq \lambda
\end{array}(13)\right. \\
\bar{F}_{\lambda}(i, j)=\frac{1}{w \times w} \sum_{m=i-w / 2}^{i+w / 2} \quad \sum_{n=j-w / 2}^{j+w / 2} F(m, n) \\
\bar{I}_{\lambda}(i, j)=1-\frac{\overline{H o}_{(i, j)-\overline{H o}_{\min }}}{\overline{H o}_{\max }-\overline{H o}_{\min }}(15)
\end{array}
$$


where $\overline{H o}(i, j)$ is the homogeneity value of $\bar{T}(\lambda)$ at $(i, j)$. w is the local window size.

After a $\lambda$-mean operation is performed on neutrosophic set $P_{N s}$, the entropy of the indeterminate subset $I$ is decreased and the distribution of the elements in $I$ becomes more non-uniform. It leads to the decrease of the indetermination of the neutrosophic set $P_{N s}$.

\subsection{Thresholding}

After the true subset $T$ is handled using $\lambda$-mean operation, the noise in $T$ is removed and $T$ becomes more homogeneous, which is suitable to segment the subset $\mathrm{T}$ precisely even using a simple threshold method.

In this paper, we utilized a simple thresholding method, Otsu's method [7] which finds the global optimum threshold $t$ that minimizes the interclass variance of the background and objects.

Otsu's method reduces the interclass variance defined by the following equation:

$$
\sigma(\mathrm{t})=\mathrm{c}_{1}(t) \sigma_{1}(\mathrm{t})+\mathrm{c}_{2}(t) \sigma_{2}(\mathrm{t})(16)
$$

where $\sigma(\mathrm{t})$ is the sum of variances of the two classes as a function of the threshold $t$. $\sigma_{i}(\mathrm{t})$ and $c_{i}(t)$ are the respective variance and probability of class $i$, $i=1,2$. Threshold $t$ that results in the minimization of $\sigma(\mathrm{t})$ separates the two classes as the foreground and background, respectively.

\section{Experiments and discussions.}

We have applied the proposed approach to a variety of images and compared the performance with that of some existing methods. A novel thresholding method [8] is based on Parzen window technique which claimed that it has the better performance on image thresholding than that of Otsu method [7], the minimum error thresholding (MET) method [9] and entropy-based method [10]. Therefore, we compared the performance of the proposed algorithm with that of the ParzenWindow based Threshloding (PWT) method [8].

\subsection{Performance evaluation}

In the experiments, we use several synthetic images to and real images to compare the performance.

An artificial image with two intensities, 0 and 255, is added with different levels of noise. Fig. 1(a) is the artificial image which was added with Guassian noise, whose mean is 0 and standard variance is 178.5. Fig. 1(b) is the thresholded result using PWT method, and Fig. 1(c) is the result by the proposed method.

It can be seen clearly that the proposed method can perform better than PWT method. Many pixels in Figs. 1(b) and 2(b) are classified into wrong groups, while they are segmented correctly in Figs. 1(c) and 2(c).

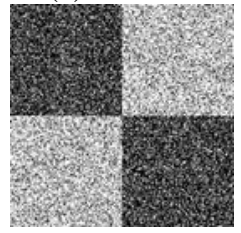

(a)

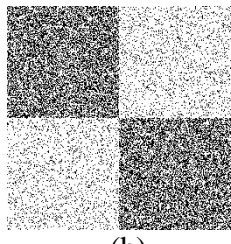

(b)

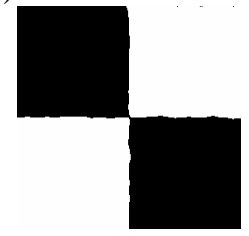

(c)
Fig. 1. (a) The image with Gaussian noise. (b)Result by the PWT method. (c)Result by the proposed method.

\subsection{Experiments on real images}

Some real images are utilized to test the performance of the proposed method. Figs. 2(a)-4(a) are the original images, Figs. 2(b)-4(b) are the results using PWT, and Figs. 2(c)-4(c) are the results using

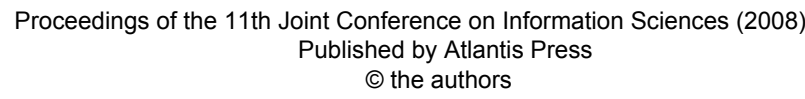


the proposed method. From the experimental results, it is shown that the thresholded images by the proposed method exhibit visually better or comparable quality.

Fig. 5(a) is the real image with Guassian noise, whose mean is 0 and standard variance is 2.55. Fig. 5(b) is the result using PWT method, which is affected deeply by the noises. The result by the proposed method in Fig. 5(c) can eliminate noise and perform better than others.

All the images in the experiments can be downloaded from: http://cvprip.cs.usu.edu/YHGuo/NSTH/Fi gures.zip .

To sum up, the proposed method is not only feasible for thresholding the clear images and but also thresholding noisy images, due to the fact that the proposed approach can handle the indetermination well.

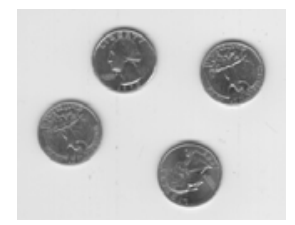

(a)

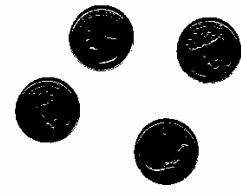

(b)

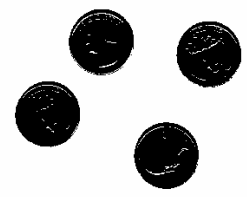

(c)
Fig. 2. (a)The original image. (b)Result by the PWT method (Threshold value: 166).

(c)Result of the proposed method (Threshold

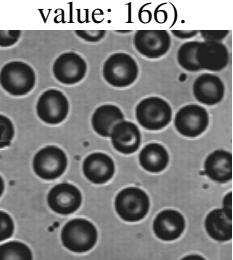

(a)

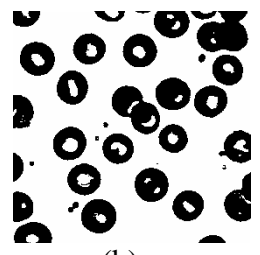

(b)

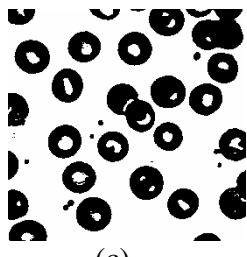

(c)
Fig. 3. (a)The original image. (b)Result by the PWT method (Threshold value: 92). (c)Result of the proposed method (Threshold value: 90 ).

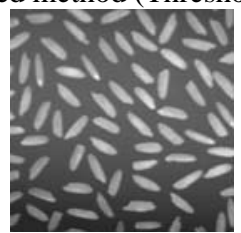

(a)

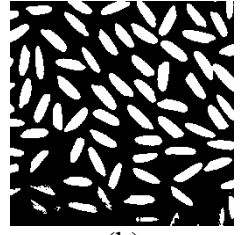

(b)

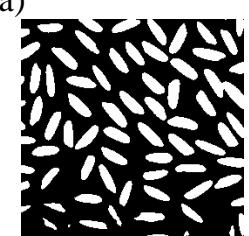

(c)
Fig. 4. (a)The original image. (b)Result by the PWT method (Threshold value: 125).

(c)Result of the proposed method (Threshold value: 120 ).

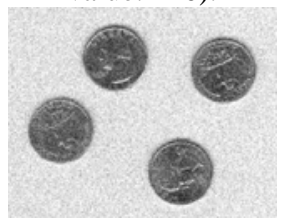

(a)

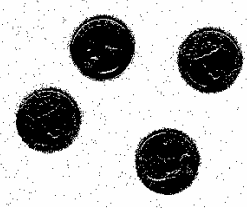

(b)

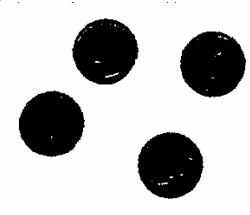

(c)
Fig. 5. (a) The original image with noise.

(b)Result by the PWT method (Threshold value: 164). (c)Result of the proposed method (Threshold value: 148).

\section{Conclusions}

In this paper, a novel neutrosophic set approach to image thresholding is proposed. The image is described as using 
three subsets. The entropy in Ns domain is defined and employed to evaluate the indetermination. A new $\lambda$-mean operation is proposed to reduce the set's indetermination. Finally, the proposed method is employed to perform thresholding. The experiment results show that the proposed method can not only perform better on clear artificial images, but also on the noisy images. The proposed approach can find more applications in image processing and patter recognition.

\section{Acknowledgement}

The work was supported, in part, by Natural Scientific Research Innovation Foundation in Harbin Institute of Technology, Project HIT.NSRIF.2008.48, and Natural Science Foundation of China No.60873142 and No. 30670546.

\section{References}

[1] F. Smarandache, A Unifying Field in Logics Neutrosophic Logic. Neutrosophy, Neutrosophic Set, Neutrosophic Probability, third ed: American Research Press, 2003.

[2] L. A. Zadeh, "Fuzzy sets," Inform and control, vol. 8, pp. 338-353, 1965.

[3] H. Wang, R. Sunderraman, F. Smarandache, and Y. Q. Zhang, Interval Neutrosophic Sets and Logic: Theory and Applications in Computing: Infinite Study, 2005.

[4] I. Turksen, "Interval valued fuzzy sets based on normal forms," Fuzzy Sets and Systems, vol. 20, pp. 191-210, 1986.

[5] K. Atanassov, "Intuitionistic fuzzy sets," Fuzzy Sets and Systems, vol. 20, pp. 87-96, 1986.

[6] K. Atanassov, "More on intuitionistic fuzzy sets," Fuzzy Sets and Systems, vol. 33, pp. 37-46, 1989.

[7] N. Otsu, "A threshold selection method from gray-level histograms," IEEE Transation on System,Man and Cybernetics, vol. 9, no. 1, pp. 62-66, 1979.

[8] S. T. Wang, F. L. Chung, and F. S. Xiong, "A novel image thresholding method based on Parzen window estimate," Pattern Recognition, vol. 41, no. 1, pp. 117-129, 2008.

[9] J. Kittler and J. Illingworth, "Minimum Error Thresholding," Pattern Recognition, vol. 19, no. 1, pp. 41-47, 1986.

[10] J. N. Kapur, P. K. Sahoo, and A. K. C. Wong, "A New Method for Gray-Level Picture Thresholding Using the Entropy of the Histogram," Computer Vision Graphics and Image Processing, vol. 29, no. 3, pp. 273285, 1985. 